\title{
Percepciones de los estudiantes de primer semestre de Medicina de sus actitudes académicas frente al curso de anatomía
}

\author{
Fabio EnRIQue Ruiz ${ }^{1}$, Michelle Cortés ${ }^{2}$
}

\section{Resumen}

Introducción. El curso de anatomía de la carrera de Medicina de la Pontificia Universidad Javeriana ofrece una metodología basada en el aprendizaje cooperativo. Para valorar las distintas actividades de dedicación personal y estudio de los estudiantes, se diseñó una autoevaluación dirigida de sus percepciones sobre sus propias actitudes con respecto al curso.

Materiales y métodos. Se diseñó un estudio descriptivo. En 2007, se aplicó a los estudiantes de primer semestre de Medicina una encuesta de autoevaluación del módulo correspondiente a la columna vertebral y al miembro superior I. El cuestionario se enfocó en la evaluación del cumplimiento de las actividades programadas, la participación del estudiante en el anfiteatro y la realización de trabajo personal. Para el procesamiento de los datos se utilizó el análisis factorial.

Resultados. Se encuestaron 78 estudiantes, con una edad promedio de 18,5 años, 50\% hombres y $50 \%$ mujeres. El análisis factorial arrojó tres factores: trabajo cooperativo, motivación intrínseca y motivación extrínseca. Las tres escalas exhibieron puntajes altos y baja variabilidad.

Discusión. El análisis de los factores evidenció que el estudiante de primer semestre de Medicina presenta una dependencia de la orientación del docente para realizar sus actividades de aprendizaje, lo que podría explicarse por la transición entre la educación secundaria y el contexto universitario que utiliza una metodología de enseñanza diferente. Asimismo, se observó que las actividades de disección y estudio en el anfiteatro requieren de un trabajo cooperativo, dada la extensión y complejidad del tema.

Palabras clave: anatomía, anfiteatro, aprendizaje, trabajo cooperativo, motivación intrínseca, motivación extrínseca, percepciones de los estudiantes.

1 Biólogo, magíster en Biología; profesor, Departamento de Morfología, Facultad de Medicina, Pontificia Universidad Javeriana, Bogotá, D.C., Colombia.

2 Médica (Universidad Nacional de Colombia), magíster en Educación; profesora, Departamento de Morfología, Facultad de Medicina, Pontificia Universidad Javeriana, Bogotá, D.C., Colombia. 


\section{Title}

Perceptions of first semester students of Medicine regarding their academic attitudes towards their anatomy module.

\begin{abstract}
Introduction: The medical degree at la Pontificia Universidad Javeriana includes an anatomy subject which offers a methodology based on collaborative learning. A supervised self assessment regarding student perceptions towards their own attitudes towards the course was developed in order to evaluate the different activities they employ for personal commitment and their methods of study.
\end{abstract}

Materials and methods: A descriptive study was designed. In 2007, a self assessment survey was given to first semester medicine students for the subject Spine and Superior Member I. The questionnaire was focused on evaluating the grade of achievement of programmed activities, evaluating student's participation at the amphitheatre and evaluating their final execution on assignments given. A factorial analysis was used to process all the data obtained.

Results: 78 students with an average age group of 18.5 years were assessed, $50 \%$ were women and $50 \%$ were men. The factorial analysis showed three factors: collaborative work, intrinsic motivation and extrinsic motivation. These three aspects showed high scores and low variability.

Discussion: The analysis revealed that first semester medicine students have a dependency on their lecturer to familiarize with the activities designed for their learning. This could be explained by the transition from secondary school to university in which students have to adjust to a different teaching methodology. Likewise, it was observed that dissection activities and amphitheatre studies require a collaborative work considering the weight and complexity given by the subject itself.

Key words Anatomy, amphitheatre, learning, collaborative work, intrinsic motivation, extrinsic motivation, student perceptions.

\section{Introducción}

En la educación superior predomina la enseñanza de contenidos disciplinarios y la evaluación de su aprendizaje suele ser la base sobre la cual se toman la mayoría de las decisiones académicas. Sin embargo, cabe resaltar que un estudiante no necesita únicamente de conocimientos para llegar a ser un profesional idóneo. El desarrollo de ciertas actitudes y comportamientos es fundamental para su adecuado desempeño en un entorno laboral que lo enfrenta a situaciones que requieren de competencias genéricas, como habilidad para trabajar en forma autónoma, capacidad para actuar en nuevas situaciones, capacidad crítica y autocrítica, capacidad de trabajo en equipo y capacidad de aprender y actualizarse permanentemente, entre muchas otras[1].

La carrera de Medicina de la Pontificia Universidad Javeriana no es ajena a estas necesidades y desde los primeros semestres se procura la adquisición de este tipo de habilidades por parte de los estudiantes. En el Departamento de Morfología, por el que pasan los estudiantes de los tres primeros semestres de Medicina, se viene implementando una metodología pedagógica dirigida hacia la adquisición tanto de conocimientos anatómicos fundamentales como de hábitos de estudio y trabajo cooperativo, indispensables para un adecuado ejercicio de la medicina. 
El curso de anatomía de primer semestre consta de cuatro módulos: generalidades de anatomía, columna vertebral y miembro superior I, miembro superior II y miembro inferior. Cada uno de estos módulos se desarrolla mediante sesiones teóricas que consisten en clases magistrales y sesiones prácticas que se llevan a cabo en el anfiteatro inmediatamente después de la sesión teórica. Para las sesiones prácticas, el grupo de estudiantes es dividido en pequeños equipos de trabajo, cada uno con un profesor tutor encargado de dirigir la disección, orientar el trabajo cooperativo, asignar tareas y evaluar el desempeño del estudiante. Además, el estudiante debe presentar, al final de cada módulo, un portafolio que recopile el trabajo realizado durante el mismo, incluyendo las tareas solicitadas por el tutor, las guías de trabajo y el atlas personal de anatomía, entre otros documentos realizados por el estudiante para favorecer su aprendizaje.

Esta metodología de trabajo intenta ofrecer al estudiante las herramientas necesarias no sólo para el aprendizaje de la anatomía, sino para el desarrollo de habilidades de comunicación efectiva y la adquisición de una actitud de reflexión y de profesionalismo. En particular, las sesiones prácticas pretenden promover en el estudiante el desarrollo de su capacidad de "aprender a aprender" en entornos cooperativos[2,3], habilidad que le será muy útil para su práctica médica en los diferentes contextos de la actuación profesional. Asimismo, la elaboración de un portafolio procura facilitar el aprendizaje del estudiante. Específicamente, el portafolio surge como una estrategia para que el estudiante tome conciencia de sus metas, progresos y dificultades, conduciéndolo a la reflexión y retroalimentación de su propio desempeño [4]. El hecho de tener que presentar un portafolio obliga al estudiante a realizar un trabajo suplementario de calidad que se convierte en una ayuda para su proceso de aprendizaje, además de ser una muy buena herramienta de autoevaluación[5].

Sin embargo, como gran parte del trabajo que el estudiante realiza durante el módulo no termina como un producto tangible y evaluable por el docente, que no necesariamente está presente en las distintas actividades de dedicación personal, estudio y trabajo cooperativo de los estudiantes, se diseñó una autoevaluación dirigida para explorar las percepciones de los estudiantes de sus propias actitudes con respecto al curso de anatomía.

\section{Materiales y métodos}

Se diseñó un estudio descriptivo exploratorio. En marzo de 2007 se aplicó a los estudiantes de primer semestre de Medicina de la Pontificia Universidad Javeriana una encuesta de 
autoevaluación del segundo módulo del curso de anatomía correspondiente a columna vertebral y miembro superior I.

Para ello, se elaboró un cuestionario autoadministrado, conformado por 13 ítems. Las preguntas se enfocaron en la evaluación del cumplimiento de las actividades programadas, la participación del estudiante en el anfiteatro y la realización de trabajo personal, utilizando una escala tipo Likert con cinco niveles de respuesta: 5 (siempre), 4 (casi siempre), 3 (algunas veces), 2 (pocas veces) y 1 (nunca). El cuestionario finalizaba con una pregunta abierta que indagaba sobre las dificultades que tuvo el estudiante durante el desarrollo del módulo. En el cuestionario se solicitó que el estudiante escribiera su nombre.

\section{Análisis estadístico}

Inicialmente, se ejecutó un análisis estadístico descriptivo de las respuestas, calculando las medias y las desviaciones estándar. Después, se realizó un análisis factorial exploratorio que se define como un conjunto de procedimientos de análisis estadístico multivariado, usados para identificar interrelaciones en un amplio grupo de variables observadas y para agrupar las variables que tienen características comunes en dimensiones o factores, mediante reducción de datos[6,7].
Este tipo de análisis estadístico le permite al investigador conceptualizar y organizar un grupo de mediciones, agrupándolas en factores que dan cuenta de ciertas dimensiones subyacentes al fenómeno observado[7]. En el caso de una encuesta, el análisis de factores enriquece la posibilidad de interpretación de los resultados frente al análisis individual de cada pregunta.

Antes de ejecutar el análisis factorial, conviene realizar ciertas pruebas para verificar que la matriz de correlaciones entre los diferentes ítems de la encuesta es adecuada para realizar el análisis[6]. La prueba de esfericidad de Bartlett determina si hay relaciones entre los ítems o si no las hay. Cuando la prueba es significativa $(p<0,05)$, se evidencia que la matriz no es una matriz identidad, es decir, que sí hay relación entre los ítems.

La medida de Kaiser-Meyer-Olkin es una medida de adecuación muestral global que muestra si hay suficiente número de datos en relación con el número de ítems para hacer el análisis factorial. Se considera adecuada si es mayor de 0,6 .

La MSA (Measurement System Analysis, MSA) es una medida de adecuación muestral individual para cada ítem y muestra si las correlaciones entre los ítems individuales son lo suficientemente fuertes como para 
sugerir la realización de un análisis factorial. Se considera adecuada si es mayor de 0,6 .

Para el análisis factorial exploratorio, se utilizó el método de extracción de cuadrados mínimos no ponderados y el método de rotación oblicua de normalización Oblimin con Kaiser. Una vez obtenidos los factores, se procedió a su interpretación. Para ello, se verificó la plausibilidad conceptual de las agrupaciones de variables sugeridas por el análisis y se determinó la dimensión correspondiente a cada factor, dándole una denominación.

Posteriormente, se crearon escalas con las variables más representativas de cada factor, se evaluó su validez con el alfa de Cronbach y se examinaron sus correlaciones utilizando el método de Pearson.

El alfa de Cronbach es una medida de confiabilidad o consistencia interna de un grupo de ítems. Representa la proporción de la varianza total de una escala que puede atribuirse a una fuente común. Para su interpretación, se considera que un valor mayor de 0,8 indica una confiabilidad buena, mientras que uno menor de 0,6 corresponde a una confiabilidad pobre[6].

La correlación de Pearson mide el grado y la dirección de la asociación entre dos variables. Su valor $r$ varía entre $-1 \mathrm{y}+1$. Los valores absolutos altos indican la existencia de una relación fuerte entre las variables, mientras que los bajos indican una asociación más débil. Los valores positivos indican una relación directa entre las variables, es decir, que si un individuo tiene un alto valor en una de las variables, tiende a tener un alto valor en la otra. Los valores negativos corresponden a una relación inversa. El grado de correlación se interpreta como sigue: débil, hasta 0,29; baja, de 0,30 a 0,49 ; moderada, de 0,50 a 0,69 ; fuerte, de 0,70 a 0,89 ; y muy fuerte, de 0,90 a 1[6]. La correlación se considera estadísticamente significativa cuando $\mathrm{p}$ es menor de 0,5 . Todos los análisis se hicieron con el programa SPSS 15.0 para Windows.

\section{Resultados}

El cuestionario fue aplicado en marzo de 2007 a 78 estudiantes de primer semestre de Medicina de la Pontificia Universidad Javeriana al finalizar el segundo módulo de anatomía. Se encuestaron 39 (50\%) hombres y $39(50 \%)$ mujeres, con una edad promedio de 18,5 años $(\mathrm{DE}=1,3)$ con un rango entre 16 y 25 años.

La mayoría de las respuestas a las preguntas oscilaron entre 4 (casi siempre) y 5 (siempre), y mostraron puntajes relativamente altos (tabla 1). El ítem 1, que indagaba sobre la asistencia, mostró el mayor puntaje pro- 
medio con una media de 4,88 ( $\mathrm{DE}=0,396)$. El $91 \%$ de los estudiantes refirió haber asistido siempre a las prácticas programadas. La pregunta con menor puntaje fue el ítem 8 , que investigaba la capacidad del estudiante de explicarle la disección a sus compañeros, con una media de 3,87 ( $\mathrm{DE}=0,843)$. El 34,6\% de los estudiantes se sintió en la capacidad de explicarle a sus compañeros sólo algunas veces y el $35,9 \%$ casi siempre. Sólo el $27 \%$ de los estudiantes respondió siempre a esta pregunta.

El estudio de la matriz de correlaciones, previo al análisis factorial, mostró una prueba de esfericidad de Bartlett significativa con una medida de Kaiser-Meyer-Olkin de 0,79. Los valores de la MSA fueron adecuados, excepto el correspondiente a la última pregunta de la encuesta (MSA=0,254), mostrando una correlación excesivamente baja con el resto de los ítems, razón por la cual esta pregunta no se incluyó dentro del análisis.

A continuación, se llevó a cabo el análisis factorial exploratorio con los 12 primeros ítems de la encuesta. Se utilizó el método de extracción de mínimos cuadrados no ponderados y el método de rotación de normalización Oblimin con Kaiser. El análisis arrojó tres factores que explicaron el 64,3\% de la varianza total. El primer factor (valor propio 4,8) explicó el 40,0\% de la varianza, el segundo factor (valor propio 1,8 ) el $14,7 \%$ y el tercer factor

Tabla 1. Resultados estadísticos descriptivos de las preguntas de la encuesta

\begin{tabular}{llrc}
\hline Ítem & & Media & DE \\
\hline 1 & ¿Asistió a todas las prácticas programadas? & 4,88 & 0,396 \\
2 & ¿Participó activamente en la disección? & 4,40 & 0,811 \\
3 & ¿Preparó sus clases con base en las guías y talleres? & 4,23 & 0,759 \\
4 & ¿Completó las guías y talleres? & 4,77 & 0,481 \\
5 & ¿Cumplió con las tareas que su tutor le asignó? & 4,72 & 0,662 \\
6 & ¿Mantuvo organizado y al día su portafolio? & 4,56 & 0,766 \\
7 & ¿Realizó tareas por iniciativa propia? & 4,08 & 1,054 \\
8 & ¿Estuvo en capacidad de explicar la disección a sus compañeros? & 3,87 & 0,843 \\
9 & ¿Colaboró con su equipo para el completo desarrollo de las tareas? & 4,21 & 0,972 \\
10 & ¿Se sintió motivado para participar en las actividades propuestas? & 4,50 & 0,734 \\
11 & ¿Le fue fácil trabajar con su equipo? & 4,65 & 0,721 \\
12 & ¿Le fue fácil trabajar con su tutor? & 4,49 & 0,802 \\
13 & ¿Comprendió la importancia de los temas vistos en este módulo & 4,83 & 0,408 \\
& para su formación profesional? & & \\
\hline
\end{tabular}

DE: desviación estándar.

Ruiz F. E., Cortés M., Percepciones de los estudiantes de primer semestre de Medicina de sus actitudes académicas... 
(valor propio 1,1) el 9,5\%. En la tabla 2 se presentan los resultados del análisis factorial; para mayor facilidad en la interpretación de los factores, se eliminaron las cargas factoriales bajas (valores absolutos menores de 0,250).

Los tres factores se definieron como sigue:

1. Trabajo cooperativo (ítems 9,10 , 11 y 12): corresponde al trabajo del estudiante con sus compañeros y con su tutor durante las prácticas de anfiteatro.

2. Motivación intrínseca (ítems 2, 3, 7 y 8): implica la realización de tareas por iniciativa propia, no exigidas por el tutor.
3. Motivación extrínseca (ítems 4, 5 y 6): incluye el cumplimiento de las tareas solicitadas por el docente durante el módulo.

Aunque algunos ítems tuvieron doble asignación a los factores, cada uno de ellos se situó en un solo factor. Para ello, se tuvieron en cuenta las cargas factoriales y la coherencia conceptual. La pregunta 1 tuvo una triple asignación con valores bajos muy cercanos entre sí, razón por la cual se decidió no asignarla a ningún factor.

A partir de cada uno de los factores descritos, se creó una escala cuyas características más sobresalientes se presentan en la tabla 3. El número de ítems se refiere a la cantidad de pre-

Tabla 2. Matriz de configuración del análisis factorial*

\begin{tabular}{llccc}
\hline & \multirow{2}{*}{$\mathbf{N}^{\circ}$ Ítem } & \multicolumn{3}{c}{ Factor** } \\
\cline { 2 - 5 } & $\mathbf{1}$ & $\mathbf{2}$ & $\mathbf{3}$ \\
\hline 11 & ¿Le fue fácil trabajar con su equipo? & 0,832 & & \\
10 & ¿Se sintió motivado para participar en las actividades propuestas? & 0,777 & & \\
12 & ¿Le fue fácil trabajar con su tutor? & 0,659 & & \\
9 & ¿Colaboró con su equipo para el completo desarrollo de las tareas? & 0,520 & 0,352 & \\
1 & ¿Asistió a todas las prácticas programadas? & 0,365 & 0,253 & 0,282 \\
8 & ¿Estuvo en capacidad de explicar la disección a sus compañeros? & & 0,741 & \\
3 & ¿Preparó sus clases con base en las guías y talleres? & & 0,622 & \\
7 & ¿Realizó tareas por iniciativa propia? & & 0,469 & \\
2 & ¿Participó activamente en la disección? & & 0,464 & \\
6 & ¿Mantuvo organizado y al día su portafolio? & & 0,828 \\
5 & ¿Cumplió con las tareas que su tutor le asignó? & & & 0,758 \\
4 & ¿Completó las guías y talleres? & & 0,630 \\
\hline
\end{tabular}

Método de extracción: mínimos cuadrados no ponderados

Método de rotación: normalización Oblimin con Kaiser

* La rotación convergió en 9 iteraciones.

** Se omiteron valores absolutos inferiores a 0,250. 
Tabla 3. Resumen de las escalas

\begin{tabular}{llcccc}
\hline Factor & Escala & $\begin{array}{c}\text { Número de } \\
\text { ítems }\end{array}$ & $\begin{array}{c}\text { Alfa de } \\
\text { Cronbach }\end{array}$ & Media & DE \\
\hline 1 & Trabajo cooperativo & 4 & 0,81 & 4,5 & 0,64 \\
2 & Motivación intrínseca & 4 & 0,68 & 4,1 & 0,61 \\
3 & Motivación extrínseca & 3 & 0,82 & 4,7 & 0,55 \\
\hline
\end{tabular}

guntas de la encuesta que se agruparon en cada escala. Las medias se presentan mediante un puntaje continuo de 1 a 5 , donde 5 corresponde a la tendencia más alta hacia dicha escala y 0 equivale a la mínima. Se examinó la validez de las escalas mediante el cálculo del alfa de Cronbach, encontrando valores aceptables en la escala "Motivación intrínseca" y buenos en las escalas "Trabajo cooperativo" y "Motivación extrínseca".

Las tres escalas exhibieron puntajes altos y baja variabilidad. La escala "motivación extrínseca" obtuvo el mayor puntaje con una media de 4,7 y la menor variabilidad con un coeficiente de variación de 0,117 .
Las correlaciones entre las escalas fueron significativas (tabla 4). Cabe resaltar la correlación positiva moderada $(\mathrm{p}<0,01)$ entre las escalas "motivación extrínseca" y "trabajo cooperativo". Esta correlación sugiere que, cuando un estudiante tenía una alta motivación extrínseca, es decir, que se interesaba por cumplir con las tareas exigidas por su tutor, tendía a trabajar de manera cooperativa, y viceversa. Asimismo, conviene señalar que la relación entre la motivación intrínseca y la extrínseca es débil, lo cual insinúa que un estudiante que se preocupaba por realizar las actividades propuestas por el docente no necesariamente tendía a desarrollar tareas por iniciativa propia, y viceversa.

Tabla 4. Correlaciones entre las escalas

\begin{tabular}{lccc}
\hline Escala & $\begin{array}{c}\text { Trabajo } \\
\text { cooperativo }\end{array}$ & $\begin{array}{c}\text { Motivación } \\
\text { intrínseca }\end{array}$ & $\begin{array}{c}\text { Motivación } \\
\text { extrínseca }\end{array}$ \\
\hline Trabajo cooperativo & 1 & $0,457(* *)$ & $0,532(* *)$ \\
Motivación intrínseca & $0,457(* *)$ & 1 & $0,280\left(^{*}\right)$ \\
Motivación extrínseca & $0,532(* *)$ & $0,280(*)$ & 1 \\
\hline
\end{tabular}

** La correlación es significativa al nivel 0,01 (bilateral)

* La correlación es significativa al nivel 0,05 (bilateral)

Ruiz F. E., Cortés M., Percepciones de los estudiantes de primer semestre de Medicina de sus actitudes académicas... 


\section{Discusión}

Se realizó un estudio descriptivo que exploró las percepciones de los estudiantes de primer semestre de Medicina de la Pontificia Universidad Javeriana sobre su desempeño al finalizar el segundo módulo del curso de anatomía. Este módulo -columna vertebral y miembro superior I- se distingue de los otros por ser el que introduce al estudiante en el trabajo en el anfiteatro. Se trata de su primera aproximación al estudio de la anatomía sobre cadáveres humanos, así como al trabajo cooperativo con sus compañeros durante las sesiones prácticas.

La realización de la encuesta no pretendió únicamente la recolección de información sobre las actitudes del estudiante; también, intentó inducirlo a un estado reflexivo sobre el cumplimiento de las distintas actividades propuestas durante el módulo, así como su compromiso con la asignatura y con sus compañeros. Los espacios para la reflexión de su propio desempeño y de su metodología de estudio durante el desarrollo del programa de anatomía, son escasos y no están formalmente establecidos. Por lo tanto, esta encuesta podría utilizarse como una herramienta pedagógica para la creación de momentos de autoevaluación inicialmente dirigidos por el docente, para que sea el estudiante quien finalmente desarrolle una capacidad autocrítica intrínseca.
En cuanto al análisis descriptivo de los ítems de la encuesta, se encontró que la pregunta sobre asistencia mostró el mayor puntaje promedio, lo que se explica fácilmente porque la asistencia al $80 \%$ de las sesiones teóricas y prácticas es un requisito indispensable para obtener una nota aprobatoria en la asignatura, según el reglamento de la universidad. En contraposición, la capacidad del estudiante para explicarle la disección a sus compañeros tuvo el menor puntaje de toda la encuesta. Esta pregunta es una de las que mejor evalúan el aprendizaje del estudiante, porque ser capaz de mostrar las estructuras halladas en la disección implica conocer la parte teórica de la anatomía, disecar y reconocer las estructuras en el cadáver y estar seguro de sus conocimientos para poder enseñarlos a otros. Esta tarea es la más difícil de cualquier módulo, pero en particular en éste, puesto que ofrece al estudiante su primer contacto con el anfiteatro.

El análisis factorial arrojó tres factores: trabajo cooperativo, motivación intrínseca y motivación extrínseca. La motivación extrínseca, que corresponde a la realización de las actividades propuestas por el docente, tuvo el mayor puntaje promedio. Por el contrario, la motivación intrínseca, que se relaciona con la propia iniciativa del estudiante, tuvo el menor puntaje. Esto indica que el estudiante de primer semestre muestra una dependencia de la orientación del docente para realizar sus 
actividades de aprendizaje. Es posible que este hallazgo se explique por las diferencias entre el entorno educativo universitario y el contexto escolar del cual proviene el estudiante. La educación secundaria se caracteriza, en general, por transmitir conocimientos vastos, presentados como completos e irrefutables, que el estudiante debe retener y memorizar, sin muchos espacios para discutir o proponer soluciones a los problemas en estudio. Esto genera en el estudiante una actitud pasiva, promoviendo una falta de interés en aprender[8]. Por el contrario, el ámbito universitario se caracteriza por tener contenidos disciplinarios específicos, amplios y profundos, que requieren un estudiante activo que discuta, critique, innove y acepte contradicciones. Por lo tanto, el primer semestre de la carrera se podría ver como una etapa de transición, en la cual el estudiante empieza a adaptarse a una nueva metodología de enseñanza.

La escala de trabajo cooperativo, es decir, el trabajo del estudiante con sus compañeros y con su tutor en las prácticas de anfiteatro, tuvo una puntuación alta. Las razones que explican este hecho pueden basarse en el ambiente de trabajo del anfiteatro, la necesidad de estudio en grupo en este contexto, la automotivación para el aprendizaje y la camaradería.

El trabajo en el anfiteatro promueve el desarrollo de actividades en gru- pos pequeños alrededor de un cadáver, lo cual genera menos tensión en los estudiantes y mayor facilidad para el establecimiento de un diálogo entre estudiantes y entre estudiantes y docentes. En efecto, la conformación de grupos pequeños de trabajo ayuda al estudiante a expresar sus ideas delante de otras personas sin temor a ser criticado o señalado[9], lo cual crea un ambiente seguro de discusión y aprendizaje. El establecimiento de este entorno cooperativo permite que los estudiantes participen activamente en su propio aprendizaje, mientras interactúan entre ellos con una mínima intervención por parte del docente[3].

Igualmente, las actividades de disección y estudio en el anfiteatro requieren de un trabajo cooperativo dada la extensión y complejidad del tema. Cuando los contenidos son amplios, el intercambio de información entre estudiantes es fundamental para mejorar su comprensión y desempeño, en particular, cuando se trata de aprender en un contexto práctico que requiere de una comprensión no sólo intelectual sino espacial en el cadáver[10]. Este acercamiento directo al objeto de estudio es muy importante para el aprendizaje, puesto que los estudiantes aprenden haciendo en un contexto pertinente, lo que proporciona un aprendizaje más significativo[11].

El aprendizaje se ve beneficiado por un entorno cooperativo que promueve 
el liderazgo de algunos estudiantes que se motivan a enseñarles a otros, reforzando así sus propios conocimientos. El aprendizaje cooperativo mejora el rendimiento académico, puesto que el intercambio de conceptos y opiniones entre los miembros del grupo promueve un aprendizaje de mayor calidad, interactivo y participativo, beneficiando a cada uno de los estudiantes[9, 12].

Finalmente, la camaradería propicia una relación cordial y de confianza entre los estudiantes, afianzando sus lazos de amistad y generando un ambiente más favorable para el aprendizaje, y facilitando el proceso de adaptación de los estudiantes al entorno de la educación superior.

Por otra parte, se encontró una correlación positiva moderada entre las escalas de motivación extrínseca y trabajo cooperativo lo cual muestra que, cuando un estudiante tiene una gran tendencia a cumplir con las tareas exigidas por su docente, también tiende a trabajar de manera cooperativa, y viceversa. Aunque no se puede hablar de causalidad, parece coherente pensar que el estudiante que tiene una alta motivación extrínseca tiende a trabajar en grupo para llevar a cabo sus tareas, lo cual confirma que las actividades propuestas al estudiante durante el módulo fomentan el desarrollo del trabajo cooperativo, uno de los propósitos consignados en la asignatura.
Las escalas creadas a partir de los factores tuvieron buenos niveles de confiabilidad, por lo que se puede decir que las preguntas agrupadas en cada una de ellas evalúan un mismo concepto. Sin embargo, estos niveles pueden y deben mejorarse para desarrollar un instrumento que brinde información más exacta y confiable con respecto al trabajo del estudiante durante el módulo. Así, con base en el análisis factorial exploratorio inicial, se podrían reescribir algunas preguntas y agregar nuevos ítems, buscando mayor especificidad en los factores. Un ejemplo de ello es el caso de la última pregunta, que no se pudo incluir dentro del análisis factorial por tener correlaciones muy bajas con el resto de los ítems de la encuesta. Esto se debe a que esta pregunta no se refiere, como las otras, a la realización de actividades o participación en las prácticas, sino a la comprensión de la importancia de los temas vistos en el módulo. Al ser un tipo de pregunta diferente a las demás, el programa estadístico no permitió incluirla en el análisis, para evitar posibles inexactitudes de agrupación durante la extracción de los factores. En consecuencia, esta pregunta debe reformularse o eliminarse de la encuesta.

Una de las limitaciones de este estudio reposa en la falta de anonimato en la encuesta. Como las preguntas examinaron la percepción del estudiante sobre su propio desempeño duran- 
te el módulo, es posible que haya un sesgo de información hacia lo positivo. Es muy probable que los estudiantes hayan reportado un desempeño superior al real, lo que puede evidenciarse con el predominio de puntajes muy altos en todas las respuestas, sin excepción.

\section{Bibliografía}

1. Beneitone P, Esquetini C, González J, Marty M, Siufi G, Wagenaar R. Reflexiones y perspectivas de la educación superior en América Latina. Informe final. Proyecto Tuning. América Latina 2004-2007. Bilbao: Universidad de Deusto. 2007.

2. Mayor J, Suengas A, González J. Estrategias metacognitivas. Madrid: Editorial Síntesis. 1995.

3. Bruffee K. Collaborative learning: higher education, interdependence, and the authority of knowledge. London: The John Hopkins University Press. 1995.

4. Monereo C, Castelló M. Las estrategias de aprendizaje. Cómo incorporarlas a la práctica educativa. Barcelona: Editorial Edebé. 1997.

5. Reyes L. Evaluación de los aprendizajes en la educación superior. Red Académica. Fecha de consulta: 10 de noviembre de 2009. Disponible en: http://www.pedagogica.edu.co/storage/ted/articulos/ted09_10arti.pdf.

6. Pett MA, Lackey NR, Sullivan JJ. Making sense of factor analysis. The use of factor analysis for instrument development in health care research. Thousand Oaks: Sage Publications, Inc. 2003.

7. Meyers LS, Gamst G, Guarino AJ. Applied multivariate research. Design and interpretation. Thousand Oaks: Sage Publications, Inc. 2006.

8. Schiefelbein E, Zúñiga R. Relaciones de la educación superior con la educación secundaria: transformación de la enseñanza, investigación y extensión universitaria. Seminario sobre Prospectiva Educativa (UNESCO). Santiago de Chile, 23-25 de agosto de 2000. Fecha de consulta: 13 de noviembre de 2009. Disponible en: http://www.schwartzman.org.br/simon/ delphi/pdf/ernesto.pdf.

9. Bernaza G, Lee F. El aprendizaje colaborativo: una vía para la educación de postgrado. Revista Iberoamericana de Educación. 2005. Fecha de consulta: 10 de noviembre de 2009. Disponible en: http://www.rieoei.org/ deloslectores/1123Bernaza.pdf.

10. Granger NA. Dissection laboratory is vital to medical gross anatomy education. Anat Rec. 2004;281B:6-8.

11. Díaz F. Cognición situada y estrategias para el aprendizaje significativo. Revista Electrónica de Investigación Educativa. 2003;5. Decha de consulta: 17 de diciembre de 2007. Disponible en: http://redie.uabc.mx/contenido/ vol5no2/contenido-arceo.pdf.

12. Johnson DW, Johnson RT, Holubec E. El aprendizaje cooperativo en el aula. Buenos Aires: Editorial Paidós. 1999. 